\title{
REDUÇÃO DO USO DE MADEIRA NO PROCESSO DE PEAÇÃO DE BOBINAS*
}

\section{Resumo}

\author{
Paulo Henrique da Silva Gomes ${ }^{1}$ \\ Eduardo Vinícius dos Santos ${ }^{2}$ \\ Gerdal de Paula Cople 3 \\ Ubaldo Marques Silva Filho ${ }^{4}$ \\ Leonardo Ribeiro Sgrancio ${ }^{5}$ \\ Luigi Guarino Neto ${ }^{6}$
}

A melhoria contínua dos processos é fator fundamental para o sucesso do negócio. Avaliar as melhores práticas e propor soluções que equilibrem os custos e o uso consciente dos recursos naturais transformará o negócio sustentável e sólido. Com esse ideal, foi realizado um estudo de viabilidade econômico-financeira e operacional para a implantação de um novo modelo de peação (fixação) de cargas conteinerizadas com produtos siderúrgicos (bobinas de aço) da CSN movimentados pelo Sepetiba Tecon - Porto de Itaguaí - RJ. O processo de peação de bobinas era basicamente realizado utilizando-se de madeiras fumigadas para a fixação das bobinas dentro do contêiner. $O$ estudo objetivou a implantação de um processo de peação utilizando cintas de poliéster, reduzindo o uso de madeira, proporcionando maior segurança pessoal e agilidade nas operações. Além disto, propôs também a utilização de um implemento para acomodações das bobinas dentro do contêiner que pode ser operado por uma empilhadeira de menor capacidade, reduzindo assim a emissão de gases poluentes e o consumo de combustíveis fósseis.

Palavras-chave: Sustentabilidade; Peação de carga; Produtos siderúrgicos.

\section{REDUCING THE USE OF WOOD IN A LASHING STEEL COILS}

\begin{abstract}
Continuous process improvement is critical to business success. Evaluate the best practices and propose solutions that balance cost and responsible use of natural resources will turn the business sustainable and solid. With this objective, an economic-financial and operational study of a new lashing model CSN steel products (steel coils) inside containers handled by Sepetiba Tecon - Itaguaí Port - RJ was conducted. The lashing coils process was basically performed using fumigated woods for fixing the coils inside the container. The study aimed to implement a process using polyester straps, reducing the use of wood, providing greater personal security and flexibility at the operations. In addition, also proposed to use a new equipment for coils accommodation that can be operated by a smaller capacity forklift, thereby reducing the emission of pollutant gaseous and the consumption of fossil fuels.
\end{abstract}

Keywords: Sustainability; Cargo lashing; Steel products.

\footnotetext{
Administrador de Empresas, Analista de Logística, CSN, Volta Redonda, RJ, Brasil.

Engenheiro de Produção, Coordenador de Logística, CSN, Volta Redonda, RJ, Brasil.

Engenheiro Civil, Gerente de Logística, CSN, Itaguaí, RJ, Brasil.

Administrador de Empresas, Gerente de Logística, CSN, São Paulo, SP, Brasil.

Administrador de Empresas, Analista de Logística, CSN, Itaguaí, RJ, Brasil.

Engenheiro Civil, Especialista de Logística, CSN, São Paulo, SP, Brasil.
}

\footnotetext{
* Contribuição técnica ao $33^{\circ}$ Seminário de Logística - Suprimentos, PCP, Transportes, 13 a 16 de maio de 2014, São Paulo, SP, Brasil.
} 


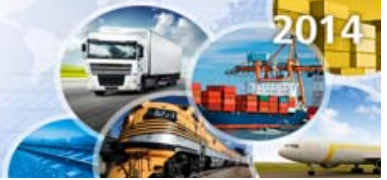

\section{INTRODUÇÃo}

O Sepetiba Tecon S. A. está localizado no Porto de Itaguaí, no Estado do Rio de Janeiro, e é um terminal marítimo responsável pela importação, exportação, transbordo e cabotagem de contêineres, produtos siderúrgicos e cargas de projeto da CSN e de terceiros. Está situado no maior polo de atividade econômica do país: $70 \%$ do PIB brasileiro têm origem em empresas instaladas em um raio de apenas 500 quilômetros [1]. De fácil acesso marítimo, rodoviário e ferroviário, é constituído de um cais contínuo de $810 \mathrm{~m}$ de comprimento, de concreto sobre estacas, ligado ao continente. A operação de cais conta com 6 portêineres e 2 MHCs (Mobile Harbour Cranes). Sua retroárea compreende, principalmente, equipamentos de pátio (RTGs,Rubber Tyred Gantry - e RSs-Reach Stackers), sistema viário interno, pátios e armazéns para estocagem de contêineres, produtos siderúrgicos e cargas de projeto e uma linha ferroviária para o carregamento e a descarga de vagões.

O Sepetiba Tecon é um dos maiores terminais de contêineres do Brasil, tendo movimentado no ano de 2013 cerca de 258 mil contêineres (380 mil TEUs - Twentyfoot Equivalent Unit). O contínuo crescimento do volume movimentado é resultante das condições geográficas, acesso rodoviário, ferroviário e aquaviário privilegiados, somados aos constantes investimentos feitos visando a uma melhoria constante do nível de serviços ofertado, buscando a excelência logística.

Durante o ano de 2012, o Sepetiba Tecon movimentou aproximadamente 2.680 contêineres com bobinas de aços da CSN. E para realizar a peação (fixação) destas bobinas o Sepetiba Tecon fez uso de empilhadeiras, madeiras e cintas metálicas.

Com o objetivo de reduzir o uso de madeiras e tornar a atividade de estufagem e peação mais segura e sustentável, foi desenvolvido um projeto que visa substituir as madeiras por cintas de poliéster e também a aquisição de um macaco hidráulico para fazer o posicionamento das bobinas dentro dos contêineres.

\section{MATERIAIS E MÉTODOS}

Este trabalho é resultado de reuniões realizadas ao longo do ano de 2013. Foram discutidas ações, ideias e planejamentos oriundos destes encontros, com participação de profissionais do setor portuário e logístico.

A metodologia adotada nestes encontros foi o Método de Melhorias PDCA [2], que é uma ferramenta da qualidade fundamentada em conceitos da administração clássica, conforme Taylor [3] e Fayol [4], os quais devem ser implementados de forma sequencial, por meio de módulos, iniciando-se pela estruturação do processo, tornando-o mensurável e repetitivo. Cada etapa está estruturada nas seguintes fases abaixo com seu grupo de atividades e o seu desenvolvimento será detalhado a seguir.

\footnotetext{
* Contribuição técnica ao 33० Seminário de Logística - Suprimentos, PCP, Transportes, 13 a 16 de maio de 2014, São Paulo, SP, Brasil.
} 



Figura 1. Ciclo PDCA.

\subsection{Processo de Estufagem e Peação de Carga}

Estufagem significa o processo de carregamento da carga junto ao contêiner. Neste processo é de suma importância não deixar espaços vazios dentro do contêiner, evitando dano às cargas e ao contêiner. Também é necessário observar o balanceamento da carga, o que dificultaria sua movimentação colocando em risco os funcionários, a carga, o navio e os equipamentos de movimentação.

Porém, quando a carga não é suficiente para preencher todos os espaços do contêiner, é necessário realizar a peação da carga (fixação), que pode ser feita através do uso de amarração com cordas, cabos, extensores, ou ser escorada por madeiras, cavaletes, estrados, bolsas de ar, ou quaisquer outros objetos que impeçam que a carga se movimente dentro do container.

A estufagem pode ser realizada manualmente, ou utilizando-se de empilhadeiras e paleteiras, ou sopradores para materiais em grãos.

\subsubsection{Operação no terminal de contêineres Sepetiba Tecon}

Durante o ano de 2012, o Sepetiba Tecon movimentou aproximadamente 2.680 contêineres com bobinas de aços da CSN, objeto desse trabalho, o que representa cerca de 8.040 bobinas (cada contêiner é carregado com 3 bobinas). Deste total de bobinas, $72 \%$ delas estavam com o seu eixo no sentido vertical ("eye to sky") e $28 \%$ com seu eixo no sentido horizontal ("eye to side").

Os processos de estufagem e peação de um contêiner de 20' supertestado com três bobinas levam, em média, 28 minutos para serem realizados demandando uma equipe de seis funcionários, quatro para a estufagem e dois para peação, e duas empilhadeiras com capacidade para cargas de 16 e 25 toneladas.

As madeiras já fumigadas (processo para eliminação de insetos e fungos conforme norma sanitária internacional) são fornecidas em peças inteiras, sendo cortadas com uso de motosserras no momento da aplicação, operação que requer cuidados especiais na prevenção de acidentes. A fixação das madeiras é realizada através do uso de martelos pneumáticos e pregos.

\footnotetext{
* Contribuição técnica ao 33 Seminário de Logística - Suprimentos, PCP, Transportes, 13 a 16 de maio de 2014, São Paulo, SP, Brasil.
} 


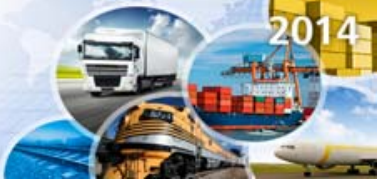

Os materiais utilizados para a peação (fixação) do contêiner estão relacionados na Tabela 1.

Tabela 1. Materiais utilizados na peação de bobinas em contêineres de 20'

\begin{tabular}{|c|c|c|}
\hline Descrição dos materiais & $\begin{array}{c}\text { Bobinas na } \\
\text { Vertical }\end{array}$ & $\begin{array}{c}\text { Bobinas na } \\
\text { Horizontal }\end{array}$ \\
\hline Palete (unid) & 3 & 3 \\
\hline Cinta metálica $(m)$ & - & 25,3 \\
\hline Selo (unid) & - & 6 \\
\hline Barrote de madeira (unid $-7,5 \times 7,5 \times 240 \mathrm{~cm}$ ) & 2 & 2 \\
\hline Barrote de madeira (unid $-7,5 \times 7,5 \times 80 \mathrm{~cm}$ ) & 6 & 6 \\
\hline Barrote de madeira (unid - 7,5 $\times 7,5 \times 40 \mathrm{~cm}$ ) & 6 & 6 \\
\hline Tábua (unid - 1' x 4' x 2,40 m) & 6 & 6 \\
\hline Cunha de madeira (unid - 7,5 $\times 7,5 \times 20 \mathrm{~cm}$ ) & 30 & 30 \\
\hline Prego (unid) & 144 & 144 \\
\hline
\end{tabular}

Considerando os volumes de contêineres movimentados com produtos siderúrgicos no ano de 2012 e a quantidade de materiais utilizados, em especial a madeira, foi identificado que o consumo anual de madeiras para realizar estas operações é da ordem de $755 \mathrm{~m}^{3}$. Esse volume é equivalente a 3.125 árvores, ou 1,16 árvores por contêiner, considerando uma árvore da espécie Eucalyptus urophylla, conhecida como Eucalipto, com o volume médio de rendimento da tora sem casca de $0,2416 \mathrm{~m}^{3}[5]$.

\subsection{Novo Processo de Peação Proposto}

Foi realizada uma pesquisa e identificado que atualmente as empresas internacionais que realizam peação de bobinas estão substituindo a utilização de madeiras por cintas de poliéster. Além de uma redução no consumo de madeira, o processo é mais rápido e de maior segurança para os funcionários, pois elimina o uso de motosserra.

Existem diversos tipos de cintas e fivelas para amarração de carga disponíveis no mercado, por isso foi necessário ainda realizar um estudo para avaliar qual equipamento se adequaria melhor às necessidades da operação.

\subsubsection{Benchmarking}

Foi realizado um mapeamento do modelo de peação de produtos siderúrgicos utilizados pelos terminais brasileiros. Após esse mapeamento foram escolhidas e realizadas visitas técnicas a quatro empresas nacionais que prestam o mesmo serviço de peação de bobinas que o Sepetiba Tecon. O objetivo era identificar as melhores práticas realizadas pelas empresas do setor e avaliar oportunidades. As quatro empresas avaliadas conforme Tabela 2 são as "big four" em sua área de atuação, e mantém operações em todos os portos brasileiros que operam produtos siderúrgicos.

Foram avaliados três quesitos nas operações: 1) Quantidade de pessoas, 2) Materiais e métodos e 3) Equipamentos. O resultado apresentado é que das quatro, apenas uma realiza o processo de peação com a utilização de cinta de poliéster e

\footnotetext{
* Contribuição técnica ao $33^{\circ}$ Seminário de Logística - Suprimentos, PCP, Transportes, 13 a 16 de maio de 2014, São Paulo, SP, Brasil.
} 
equipamento diferenciado na estufagem de contêineres. As demais utilizam o mesmo método e materiais em seus processos quando comparados ao Sepetiba Tecon com a mesma quantidade de pessoas e equipamentos envolvidos.

Tabela 2. Comparativo Operacional das big four do setor

\begin{tabular}{ccll}
\hline Empresa & Equipe & Equipamentos & $\begin{array}{l}\text { Materiais e } \\
\text { Métodos }\end{array}$ \\
\hline A & 6 pessoas & Macaco hidráulico + empilhadeira & pequena \\
B & 6 pessoas & 2 empinta Poliéster \\
C & 6 pessoas & 2 empilhadeiras grandes & Madeira \\
D & 6 pessoas & 2 empilhadeiras grandes & Madeira \\
\hline
\end{tabular}

Foi observado que o processo de estufagem e peação de bobinas em contêineres com o uso de cinta de poliéster, não altera a quantidade de bobinas por contêiner pois o determinante nesse tema é o peso máximo permitido, que é pouco impactado com a redução do uso da madeira. A quantidade de pessoas envolvidas na operação é a mesma do processo atual.

O tempo de operação observado na empresa "A" foi de aproximadamente 15 minutos contra os 28 minutos realizados pelo Sepetiba Tecon, devido a maior flexibilidade e facilidade do processo. Isso representa uma redução de $46,4 \%$ no tempo do processo de estufagem e peação além de eliminar completamente o uso de motosserra para corte das madeiras. As cunhas que permanecem no processo de peação já vêm prontas para uso do fornecedor. A metodologia de aplicação é de fácil assimilação e reduz consideravelmente os riscos de acidentes pessoais quando comparado ao processo atual.

Observou-se também uma redução na quantidade de materiais envolvidos no processo, conforme Tabela 3.

Tabela 3. Materiais utilizados na peação de bobinas em contêineres de 20'.

\begin{tabular}{lcc}
\hline Descrição dos materiais & $\begin{array}{c}\text { Bobinas na } \\
\text { Vertical }\end{array}$ & $\begin{array}{c}\text { Bobinas na } \\
\text { Horizontal }\end{array}$ \\
\hline Cinta de amarração $(\mathrm{m})$ & 18 & 18 \\
Fivela (unid) & 6 & 6 \\
Cunha de madeira (unid $-7,5 \times 7,5 \times 20 \mathrm{~cm}$ ) & 24 & 24 \\
Prego (unid) & 78 & 78 \\
\hline
\end{tabular}

Além dos pontos descritos acima, observou-se também que para a movimentação e acomodação das bobinas dentro do contêiner foi utilizado um macaco hidráulico acoplado a uma empilhadeira de pequeno porte, mais ágil e de menor consumo de combustível, com capacidade para carga de até 6 toneladas.

\subsubsection{Macaco hidráulico}

O macaco hidráulico observado na visita realizada na empresa " $A$ " tem por aplicação movimentar bobinas de aço nas posições vertical e horizontal, com capacidade de carga de até 20 toneladas.

Este equipamento é composto de estrutura móvel de aço sobre pneus maciços superelásticos para elevação da carga através de sistema hidráulico, cambão medindo 6 metros, aríete compatível e contrapeso. Este conjunto deve ser acoplado

\footnotetext{
* Contribuição técnica ao $33^{\circ}$ Seminário de Logística - Suprimentos, PCP, Transportes, 13 a 16 de maio de 2014, São Paulo, SP, Brasil.
} 


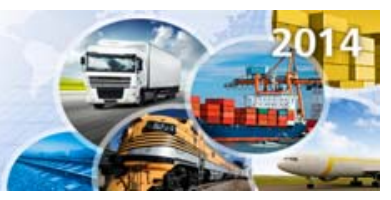

através de tomada hidráulica de engate rápido a um comando extra da empilhadeira, sendo compatível com empilhadeiras de 4 a 7 toneladas. A Figura 2 ilustra 0 equipamento utilizado.

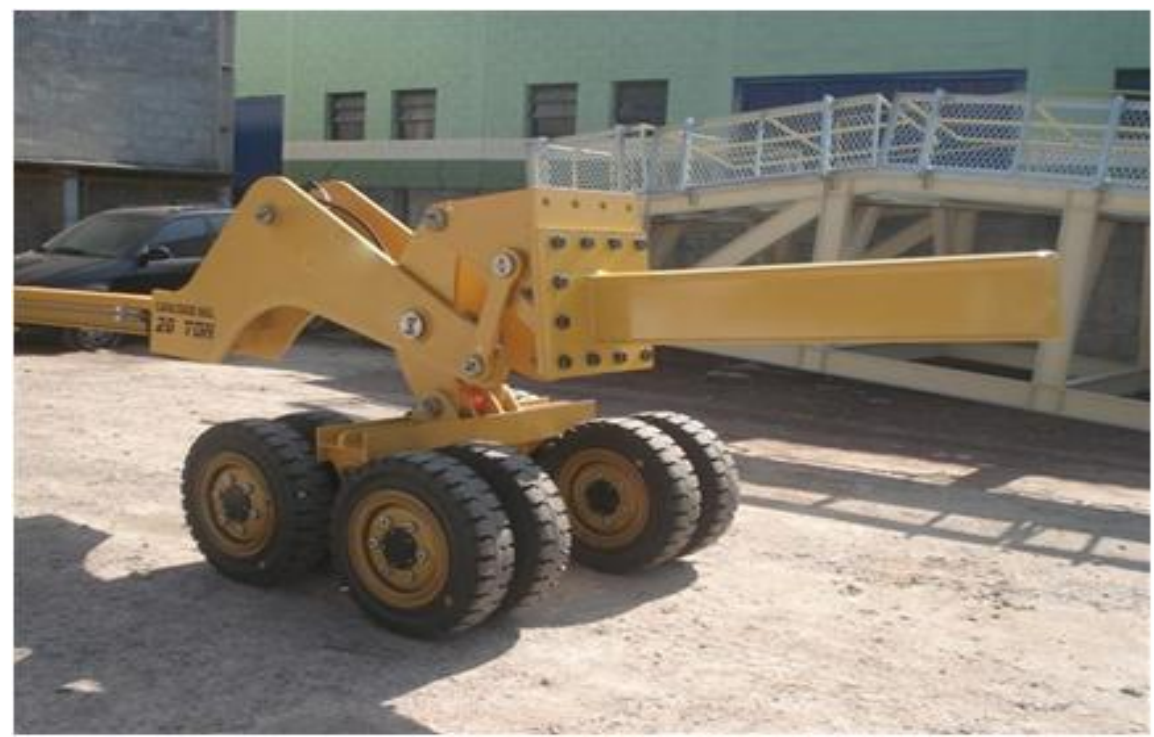

Figura 2. Macaco hidráulico.

\section{RESULTADOS E DISCUSSÃO}

Comparando os processos aplicados pelo Sepetiba Tecon e os processos aplicados pela empresa " $A$ " visitada, uma vez que as demais mantém o mesmo padrão operacional utilizado pelo Sepetiba Tecon, observam-se ganhos significativos em vários aspectos na proposta de alteração do modelo para uso de cinta de poliéster.

\subsection{Tempo de Operação}

Com a utilização do macaco hidráulico e a substituição de madeiras por cintas de poliéster para a peação da carga, o tempo de estufagem e peação cai de 28 minutos por contêiner para 15 minutos por contêiner, uma queda de 46,4\%. Isso representa um aumento de produtividade da equipe, quase dobrando a quantidade diária de contêineres estufados/dia.

\subsection{Consumo de Madeiras}

Aplicando-se o modelo de peação de carga com cintas de poliéster, tem-se uma redução de 50,0\% do consumo de madeira, isto significa que mais de 1.560 árvores por ano deixarão de serem cortadas. Além da substituição de barrotes e cunhas por cintas de poliéster, com o macaco hidráulico é possível acomodar as bobinas com eixo na posição horizontal sem a utilização de paletes. Serão utilizadas apenas tábuas para escoramento sem afetar a segurança da carga, do contêiner e dos funcionários.

\footnotetext{
* Contribuição técnica ao $33^{\circ}$ Seminário de Logística - Suprimentos, PCP, Transportes, 13 a 16 de maio de 2014, São Paulo, SP, Brasil.
} 
Tabela 4. Comparativo de utilização de materiais entre a peação modelo anterior e novo em contêineres de 20

\begin{tabular}{|c|c|c|c|c|}
\hline \multirow[b]{2}{*}{ Descrição dos materiais } & \multicolumn{2}{|c|}{ MODELO ANTERIOR } & \multicolumn{2}{|c|}{ NOVO MODELO } \\
\hline & $\begin{array}{c}\text { Bobinas na } \\
\text { Vertical } \\
\end{array}$ & $\begin{array}{c}\text { Bobinas na } \\
\text { Horizontal } \\
\end{array}$ & $\begin{array}{c}\text { Bobinas na } \\
\text { Vertical }\end{array}$ & $\begin{array}{c}\text { Bobinas na } \\
\text { Horizontal } \\
\end{array}$ \\
\hline Palete (unid) & 3 & 3 & 3 & - \\
\hline Cinta metálica (m) & - & 25,3 & - & - \\
\hline Selo (unid) & - & 6 & - & - \\
\hline Barrote (unid - 7,5 x 7,5 x $240 \mathrm{~cm}$ ) & 2 & 2 & - & - \\
\hline Barrote (unid - 7,5 $\times 7,5 \times 80 \mathrm{~cm}$ ) & 6 & 6 & - & - \\
\hline Barrote (unid - 7,5 x 7,5 x $40 \mathrm{~cm}$ ) & 6 & 6 & - & - \\
\hline Tábua (unid - 1'x 4' x $240 \mathrm{~cm}$ ) & 6 & 6 & - & 6 \\
\hline Cunha (unid-7,5 $\times 7,5 \times 20 \mathrm{~cm}$ ) & 30 & 30 & 24 & 24 \\
\hline Prego (unid) & 144 & 144 & 54 & 78 \\
\hline Cinta de Poliester $(m)$ & - & - & 18 & 18 \\
\hline Fivela (unid) & - & - & 6 & 6 \\
\hline TOTAL MADEIRAS $\left(\boldsymbol{m}^{3}\right)$ & 0,282 & 0,282 & 0,171 & 0,064 \\
\hline
\end{tabular}

Além da redução do consumo de madeira, há também uma melhora na eficiência enérgica e redução de emissão de gases poluentes durante o novo processo, pois com a utilização do macaco hidráulico, não há a necessidade de utilização de duas empilhadeiras de grande porte movidas à diesel, podendo-se utilizar apenas uma empilhadeira de pequeno porte movida à gás para a realização de todo o processo de estufagem e peação.

Também foi desenvolvida uma tabela de aspectos e impactos ambientais, que confirma que o uso de cinta de poliéster é menos nocivo ao meio ambiente: 
Tabela 5. Tabela de Aspectos e Impactos Ambientais

\begin{tabular}{|c|c|c|c|c|c|c|c|c|}
\hline $\begin{array}{l}\frac{\pi}{\frac{\pi}{\pi}} \\
\frac{\pi}{\sigma} \\
\vdash\end{array}$ & Aspecto Ambiental & Impacto Ambiental & 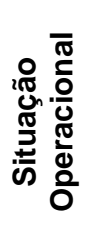 & $\begin{array}{l}\frac{0}{0} \\
\frac{\pi}{0} \\
\frac{0}{\sigma} \\
\frac{0}{0} \\
\frac{0}{\varepsilon} \\
\stackrel{0}{上}\end{array}$ & $\begin{array}{l}\frac{0}{0} \\
\frac{0}{2} \\
\frac{5}{0} \\
\frac{1}{\Sigma}\end{array}$ & 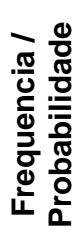 & $\begin{array}{l}\frac{\pi}{0} \\
\frac{c}{\pi} \\
\frac{t}{0} \\
\frac{0}{\Xi}\end{array}$ & 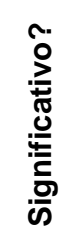 \\
\hline \multirow{12}{*}{ 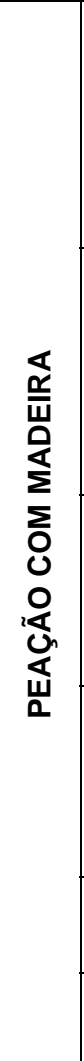 } & \multirow{3}{*}{$\begin{array}{l}\mathrm{RN}-02 \text { - Consumo de } \\
\text { recursos florestais nativos } \\
\text { (ex.:madeira, carvão } \\
\text { vegetal) }\end{array}$} & $\begin{array}{l}\text { IRN-04 - Redução da } \\
\text { disponibilidade de } \\
\text { recursos naturais e/ou } \\
\text { energia. }\end{array}$ & $\mathrm{R}$ & $A$ & 1 & 3 & 3 & NÃO \\
\hline & & IF-01 - Danos à flora & $\mathrm{R}$ & $A$ & 1 & 3 & 3 & NÃO \\
\hline & & IF-02 - Danos à fauna & $\mathrm{R}$ & $A$ & 1 & 3 & 3 & NÃO \\
\hline & \multirow{3}{*}{$\begin{array}{l}\text { RS-05 - Geração de } \\
\text { Resíduos - Madeira }\end{array}$} & $\begin{array}{l}\text { IRN-04 - Redução da } \\
\text { disponibilidade de } \\
\text { recursos naturais e/ou } \\
\text { energia. }\end{array}$ & $\mathrm{N}$ & A & 1 & 3 & 3 & NÃO \\
\hline & & IF-01 - Danos à flora & $\mathrm{N}$ & $A$ & 1 & 3 & 3 & NÃO \\
\hline & & IF-02 - Danos à fauna & $\mathrm{N}$ & $A$ & 1 & 3 & 3 & NÃO \\
\hline & \multirow{2}{*}{$\begin{array}{l}\text { EA-02 - Emissão de gases } \\
\text { de combustão (SOX, NOX, } \\
\text { CO, CO2) }\end{array}$} & $\begin{array}{l}\text { IA-02 - Alteração da } \\
\text { camada de ozônio }\end{array}$ & $\mathrm{N}$ & A & 1 & 3 & 3 & NÃO \\
\hline & & $\begin{array}{l}\text { IA-04 - Agravamento do } \\
\text { efeito estufa }\end{array}$ & $\mathrm{N}$ & A & 1 & 3 & 3 & NÃO \\
\hline & \multirow{2}{*}{$\begin{array}{l}\text { RN-01 - Consumo de } \\
\text { combustíveis fósseis }\end{array}$} & $\begin{array}{l}\text { IA-02 - Alteração da } \\
\text { camada de ozônio }\end{array}$ & $\mathrm{N}$ & $A$ & 1 & 3 & 3 & NÃO \\
\hline & & $\begin{array}{l}\text { IA-04 - Agravamento do } \\
\text { efeito estufa }\end{array}$ & $\mathrm{N}$ & $A$ & 1 & 3 & 3 & NÃO \\
\hline & $\begin{array}{l}\text { EA-01 - Emissão de } \\
\text { material particulado }\end{array}$ & IRN-03 - Dano Ambiental & $\mathrm{N}$ & $A$ & 1 & 3 & 3 & NÃO \\
\hline & $\begin{array}{l}\text { RI-06 - Queda de Material } \\
\text { (diversos) ao mar }\end{array}$ & IRN-03 - Dano Ambiental & $\mathrm{R}$ & $A$ & 1 & 3 & 3 & NÃO \\
\hline \multirow{5}{*}{ 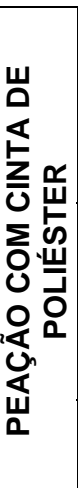 } & \multirow[t]{2}{*}{$\begin{array}{l}\text { RS-10 - Geração de } \\
\text { Resíduos - } \\
\text { Plástico/Polímeros } \\
\text { Sintéticos }\end{array}$} & $\begin{array}{l}\text { IRN-04 - Redução da } \\
\text { disponibilidade de } \\
\text { recursos naturais e/ou } \\
\text { energia. }\end{array}$ & $\mathrm{N}$ & $F$ & 1 & 3 & 3 & NÃO \\
\hline & & IRN-03 - Dano Ambiental & $\mathrm{N}$ & $\mathrm{F}$ & 1 & 3 & 3 & NÃO \\
\hline & \multirow[t]{2}{*}{$\begin{array}{l}\text { RS-06 - Geração de } \\
\text { Resíduos Metálicos }\end{array}$} & $\begin{array}{l}\text { IRN-04 - Redução da } \\
\text { disponibilidade de } \\
\text { recursos naturais e/ou } \\
\text { energia. }\end{array}$ & $\mathrm{N}$ & $\mathrm{F}$ & 1 & 3 & 3 & NÃO \\
\hline & & IRN-03 - Dano Ambiental & $\mathrm{N}$ & $\mathrm{F}$ & 1 & 3 & 3 & NÃO \\
\hline & $\begin{array}{l}\text { RI-06 - Queda de Material } \\
\text { (diversos) ao mar }\end{array}$ & IRN-03 - Dano Ambiental & $\mathrm{R}$ & $\mathrm{F}$ & 1 & 3 & 3 & NÃO \\
\hline
\end{tabular}

Legenda: $\boldsymbol{R}$ - Risco / N - Normal / A - Atual / F - Futuro / 1 - Baixo / 2 - Médio / 3 - Alto

\subsection{Custo Operacional}

Durante a avaliação técnica realizada na empresa "A" foi observado que a cinta de poliéster utilizada no processo estava superdimensionada. Usava-se uma cinta de $40 \mathrm{~mm}$ admitindo uma resistência de até $8.500 \mathrm{kgf}$. Uma vez que o olhal de um contêiner (olhal é um ponto dentro do contêiner onde é feita a amarração das cintas de poliéster) possui uma resistência de até $2.600 \mathrm{kgf}$, a capacidade da cinta empregada no processo era 3 vezes superior à capacidade do olhal. Uma vez observado a oportunidade de adequação da cinta, procurou-se no mercado uma

\footnotetext{
* Contribuição técnica ao $33^{\circ}$ Seminário de Logística - Suprimentos, PCP, Transportes, 13 a 16 de maio de 2014, São Paulo, SP, Brasil.
} 
cinta de poliéster com menor resistência e que fosse compatível com a aplicação necessária. Foi encontrada uma cinta de poliéster de $32 \mathrm{~mm}$, com uma resistência de $2.620 \mathrm{kgf}$, adequada as especificações com certificação de resistência necessária. A simples substituição de uma cinta de $40 \mathrm{~mm}$ por uma cinta de $32 \mathrm{~mm}$ gerou uma redução de custos com o material na ordem de $60 \%$ por contêiner.

Portanto, aplicando-se este novo modelo de peação de carga, tem-se uma redução dos custos operacionais totais de $27 \%$ e $69 \%$ para as bobinas na vertical e na horizontal, respectivamente. A redução de custos pode ser de até $49 \%$ ao ano, baseado nas movimentações de contêineres de 2012.

\section{CONCLUSÃO}

A aplicação de cintas de poliéster em substituição ao uso de madeiras e a utilização do macaco hidráulico geram resultados economicamente viáveis e ajudam na preservação de recursos naturais, dando sustentabilidade ao negócio. Além disso, dão condições mais seguras para os funcionários envolvidos no processo, garantindo um melhor manuseio da carga e conservação dos contêineres (assoalho).

A CSN tem por objetivo, mediante aos resultados deste projeto, aplicar esta metodologia operacional para outros tipos de cargas siderúrgicas, como por exemplo, os fardos de folhas metálicas, que não fizeram parte do escopo inicial do projeto.

\section{REFERÊNCIAS}

1 IBGE: Instituto Brasileiro de Geografia e Estatística [página da internet]. Brasil, 2013 [acesso em 04 fev. 2014]. Disponível em:

http://www.ibge.gov.br/home/estatistica/economia/pibmunicipios/2011/default_pdf.shtm. Andrade FF. O método de melhorias PDCA [dissertação de mestrado em Engenharia de Construção Civil e Urbana] . São Paulo: Escola Politécnica, Universidade de São Paulo; 2003.

3 Taylor FW. Princípios de administração científica. 8ª ed. São Paulo: Atlas; 1990.

4 Fayol H. Administração industrial e geral: previsão, organização, comando, coordenação, controle. $10^{\text {a }}$ ed. São Paulo: Atlas; 1989.

5 Scanavaca Junior L, Garcia JN. Rendimento em madeira serrada de Eucalyptus urophylla. In: Scientia Forestalis. Instituto de Pesquisas e Estudos Florestais. n.50. Piracicaba: IPEF; 1996. p.32-43. 